\title{
Magnétisme et matériaux magnétiques : introduction
}

\section{J. DEGAUQUE}

Laboratoire de Physique des Solides, associé au CNRS, I.N.S.A., Complexe Scientifique de Rangueilh 31077 Toulouse cedex, France

\begin{abstract}
This paper reviews a few main aspects of the solid state magnetism necessary to understand magnetic properties and behaviour of magnetic materials. The treatment of a subject as broad as present one must necessarily be limited and incomplete. Netherless we deal with the role of magnetism and magnetic materials in modern technology and cover some aspects of material such as : magnetostrictive and magnetoresistive materials, soft and hard magnetic materials, magnetic multilayers and magnetic recording.
\end{abstract}

\section{INTRODUCTION}

Du fait de leur nombreuses applications technologiques, les matériaux magnétiques ont, à l'échelle mondiale une importance économique comparable à celle des semiconducteurs. Les recherches entreprises ces dernières années et activement poursuivies aujourd'hui dans les laboratoires des pays industrialisés ont permis de réaliser la synthèse de nouveaux matériaux magnétiques aux performances toujours plus élevées. Le but de cet article est de rappeler quelques uns des phénomènes gouverıant le magnétisme des solides et d'illustrer leur influence sur certaines caractéristiques de quatre grands groupes de matériaux magnétiques :

- les trois premiers groupes qui constituent les matériaux magnétiques "classiques", parce que connus depuis longtemps, si ce n'est qu'ils sont en perpétuelle mutation. Il s'agit des matériaux magnétiques "doux", "durs" -ou matériaux pour aimants permar $n$ nts- et des matériaux destinés à l'enregistrement magnétique ;

- le quatrième groupe, qui mérite vraiment le qualificatif de "matériaux nouveaux", comprend les super réseaux métalliques magnétiques qui ont vu le jour au milieu des années 1980. 


\section{ORIGINE DU MAGNETISME D'UNE ASSEMBLEE D'ATOMES}

Présentent un moment magnétique, les atomes qui posssèdent une couche électronique incomplète (couche $3 \mathrm{~d}$ des métaux de transition (M.T) et couche $4 \mathrm{f}$ des métaux de terres rares (T.R)). Le moment magnétique atomique provient alors :

- de la contribution orbitale de moment magnétique $\vec{\mu}_{L}$, due à la rotation des électrons autour du noyau.

- de la contribution de spin de moment magnétique $\vec{\mu}_{s}$

Dans la matière condensée, le magnétisme résulte (fig. 1) :

- d'un ensemble de moments magnétiques de spins couplés entre eux par des interactions d'échange $E_{e}$, d'origine électrostatique et de très courte portée. Lorsque l'énergie d'échange $E_{e}$ est supérieure à l'activation thermique, et selon son signe, on observe un ordre ferromagnétique ( $\mathrm{E}_{\mathrm{e}}>0$ : les moments $\vec{\mu}_{s}$ sont parallèles entre eux), un ordre antiferromagnétique ( $\mathrm{E}_{\mathrm{e}}<0$ : le moments sont antiparallèles et se compensent exactement) ou un ordre ferrimagnétique $\left(\mathrm{E}_{\mathrm{e}}<0\right.$ : existence de deux types de porteurs différents couplés antiparallèlement et appartenant à deux sous réseaux distincts; il subsiste un moment magnétique résultant)

- d'un ensemble de moments magnétiques orbitaux couplés au réseau cristallin par l'intermédiaire du champ cristallin $\mathrm{E}_{\text {crist }}$ induit par les charges ioniques de l'environnement et qui est à l'origine de l'anisotropie magnétocristalline $\mathrm{E}_{\mathbf{K}}$

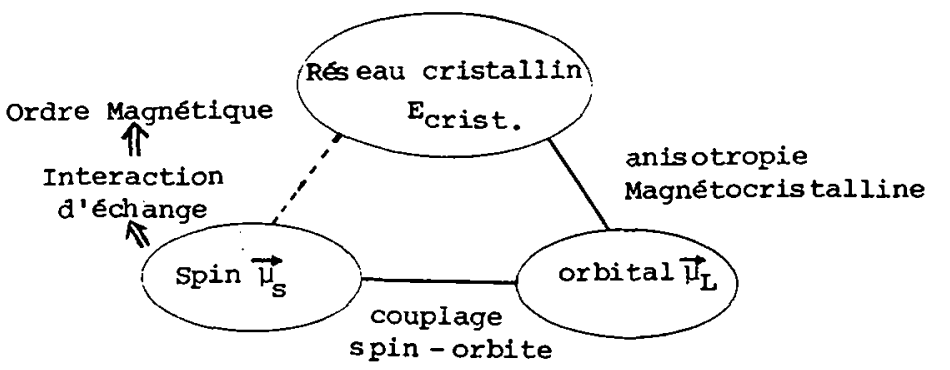

Figure 1 : Principales contributions du magnétisme dans la matière condensée

- d'une interaction entre ces deux ensembles, le couplage spin-orbite $E_{L} . S$, d'origine relativiste qui tend à aligner les moments magnétiques orbitaux parallèlement aux moments de spins afin de donner le moment résultant $\vec{\mu}_{M}$

\section{EFFETS DE LA LOCALISATION DES COUCHES ELECTRONIQUES INCOMPLETES}

Dans les métaux de transition, la couche $3 d$ est une couche externe : les interactions d'échange sont directes et très importantes. Il en résulte que l'ordre ferromagnétique subsiste jusqu'à des températures relativement élevées, de l'ordre de $1000 \mathrm{~K}\left(\mathrm{Fe}: \mathrm{T}_{\mathrm{c}}=1043 \mathrm{~K} ; \mathrm{Co}\right.$ : $\mathrm{T}_{\mathrm{c}}=1388 \mathrm{~K} ; \mathrm{Ni}: \mathrm{T}_{\mathrm{c}}=627 \mathrm{~K}$ ). Les orbites électroniques sont alors très fortement couplées au réseau cristallin ( $\mathrm{E}_{\text {crist. }}>\mathrm{E}_{\mathrm{L} . \mathrm{S}}$, de un à deux ordres de grandeur) ce qui réduit nettement le moment orbital : l'anisotropie magnétocristalline $\mathrm{E}_{\mathrm{K}}$ est relativement faible (la principale constante d'anisotropie $\mathrm{K}_{1}\left(\mathrm{~J} \cdot \mathrm{m}^{-3}\right)$ pour $\left.\mathrm{Ni}:-5,1 \times 10^{3}, \mathrm{Fe}: 4,7 \times 10^{4} ; \mathrm{Co}: 4,1 \times 10^{5}\right)$ ce qui est mis à profit pour réaliser des matériaux magnétiques "doux", c'est-à-dire présentant une 
inversion aisée de l'aimantation globale. Au contraire dans les métaux de terres rares, la couche $4 \mathrm{f}$ est interne et partiellement écrantée par les couches $5 \mathrm{~s}$ et $5 \mathrm{p}$. Il en résulte des interactions d'échange indirectes, transmises par les électrons de conduction: l'ordre ferromagnétique n'apparaît qu'au-dessous de la température ambiante (Gd: $T_{c}=293 \mathrm{~K}, \mathrm{~Tb}$ : $\left.\mathrm{T}_{\mathrm{c}}=220 \mathrm{~K} ; \mathrm{Dy}: \mathrm{T}_{\mathrm{c}}=85 \mathrm{~K}\right)$. Le couplage spin-orbite est important $\left(\mathrm{E}_{\text {cris. }}<\mathrm{E}_{\mathrm{L} . \mathrm{S} \text {, d'un }}\right.$ ordre de grandeur environ) ce qui permet aux moments orbitaux d'être davantage liés aux moments de spin qu'au réseau cristallin : l'anisotropie magnétocristalline peut être de deux ordres de grandeurs supérieure $\left(\mathrm{K}_{1} \approx 10^{6}\right.$ à $\left.10^{7} \mathrm{~J} . \mathrm{m}^{-3}\right)$ à celle des M.T.

L'énergie magnétocristalline est minimale lorsque les moments magnétiques résultant $\vec{\mu}_{M}$ sont dirigés selon les directions de facile aimantation. Dans le cas des M.T, de structure cubique Fe $\left(C . C ; K_{1}>0\right)$ et $\mathrm{Ni}\left(C . F . C ; K_{1}<0\right)$ ce sont respectivement les directions $<100>$ et $\left\langle 111>\right.$. Pour $\mathrm{Co}\left(\mathrm{H} . \mathrm{C} ; \mathrm{K}_{1}>0\right)$ la direction facile est celle de l'axe $\vec{c}$. Pour les métaux T.R, la compétition entre l'énergie d'échange et l'énergie d'anisotropie peut conduire à des configurations complexes des moments magnétiques (structures hélimagnétiques, modulées ...).

D'un point de vue pratique, la synthèse de composés de terres rares riches en métaux de transition peut conduire à des matériaux magnétiques performants caractérisés par une température de Curie et des constantes d'anisotropie élevées. Ainsi les aimants permanents les plus puissants actuellement connus sont obtenus à partir de composés intermétalliques : T.R + M.T, soit : $\mathrm{Sm}_{2} \mathrm{Co}_{17}\left(\mathrm{~K}_{1}=0,3.10^{7} \mathrm{~J} \cdot \mathrm{m}^{-3}\right), \mathrm{SmCo}_{5}\left(\mathrm{~K}_{1}=1,1.10^{7} \mathrm{~J} \cdot \mathrm{m}^{-3}\right)$ et $\mathrm{Nd}_{2} \mathrm{Fe}_{14} \mathrm{~B}\left(\mathrm{~K}_{1}=\right.$ $1,7 \cdot 10^{7} \mathrm{~J} \cdot \mathrm{m}^{-3}$ ).

Examinons deux conséquences de couplage spin-orbite :

Tableau 1: Valeurs des coefficients de magnétostriction

$\left(\times 10^{-6}\right)$ de métaux cubiques, dans les directions $<100\rangle,\langle 111\rangle$ et pour un polycristal

\begin{tabular}{|l|l|l|l|}
\hline $\begin{array}{l}\text { Métaux } \\
\text { cubiques }\end{array}$ & $\lambda_{100}$ & $\lambda_{\text {III }}$ & $\lambda_{\text {poly }}$ \\
\hline $\mathrm{Fe}$ & 21 & -21 & -7 \\
$\mathrm{Ni}$ & -46 & -24 & -34 \\
$\mathrm{FeOFe}_{2} \mathrm{O}_{3}$ & -20 & 78 & 40 \\
\hline $\mathrm{Tb}_{0.27} \mathrm{Dy}_{0.73} \mathrm{Fe}_{1.95}$ & 90 & 1600 & 1495 \\
\hline
\end{tabular}

\section{- La magnétostriction spontanée et à} saturation.

Lorsqu'on refroidit un matériau ferromagnétique au-dessous de son point de Curie, il apparait une déformation spontanée du réseau due à l'ordre magnétique qui induit, par l'intermédiaire du couplage spin-orbite, une déformation des orbitales électroniques : c'est la magnétostriction spontanée. Le matériau soumis à un champ magnétique peut alors se déformer donnant lieu à la magnétostriction linéaire que l'on caractérise par

l'allongement relatif $\lambda=\Delta \ell / \ell$ et qui tend vers une valeur limite à saturation $\lambda_{s}$ pour les forts champs. Dans le cas des métaux de transition, le moment magnétique orbital étant pratiquement bloqué en présence d'un couplage spin-orbite relativement réduit, l'orientation des spins dans la direction du champ n'a que peu d'influence sur les déformations : les valeurs de la magnétostriction sont modestes et anisotropes (tableau I).

Au contraire, dans le cas des métaux de terres rares, le moment magnétique orbital étant peu bloqué, on peut observer des valeurs élevées de la magnétostriction à saturation. Par exemple, le composé monocristallin DyZn $\left(\mathrm{T}_{\mathrm{c}}=140 \mathrm{~K}\right)$ présente à $4,2 \mathrm{~K}$ une magnétostriction "géante" et record $\lambda_{s}=5300 \times 10^{6}[1]$. D'un point de vue plus pratique, le terfenol $\mathrm{TbFe}_{2}\left(\mathrm{~T}_{\mathrm{c}} \approx\right.$ 
$698 \mathrm{~K}$ ) et le terfenol-D $\mathrm{Tb}_{0.3} \mathrm{Dy}_{0.7} \mathrm{Fe}_{2}$ présentent à $300 \mathrm{~K}$ des coefficients de magnétostriction $\lambda_{s}$ élevés respectivement voisins de $1750 \times 10^{-6}$ et $1100 \times 10^{-6}$, ce qui est mis à profit pour la réalisation d'émetteurs et récepteurs acoustiques, d'actionneurs ...

L'effet Villari constitue l'effet réciproque de la magnétostriction à savoir qu'une contrainte mécanique $\sigma$ de tension (compression) appliquée à un matériau présentant une magnétostriction positive (négative) tend à orienter les moments magnétiques parallèlement à $\sigma$. Dans le cas très simplifié d'une magnétostriction supposée isotrope $\lambda_{100}=\lambda_{111}=\lambda_{s}$, il apparaît alors une énergie magnétoélastique $E_{\lambda} \sim 3 / 2 \lambda_{s} \sigma$

\section{- La magnétorésistance spontanée :}

La magnétorésistance spontanée est due à la variation de résistivité résultant de la déformation du nuage électronique de chaque atome [2]. Dans un métal ferromagnétique parcouru par un courant uniforme, on définit par $\rho_{/,}$et $\rho_{1}$ les résistivités mesurées lorsque le courant est respectivement parallèle et perpendiculaire à l'aimantation, supposée orientée par l'application d'un champ magnétique. Généralement, on vérifie que $\rho_{/ /}>\rho_{1}$. L'anisotropie de la résistivité et le rapport de magnétorésistance sont respectivement définis par :

$$
\Delta \rho=\rho_{1 /}-\rho_{\perp} \quad \text { et } \quad \frac{\Delta \rho}{\rho}=\frac{\rho_{1 /} \rho_{1}}{\rho}
$$

où $\rho$ est la résistivité de l'échantillon désaimanté. Tout comme la magnétostriction, la magnétorésistance est liée à la symétrie cristalline (fig. 2).
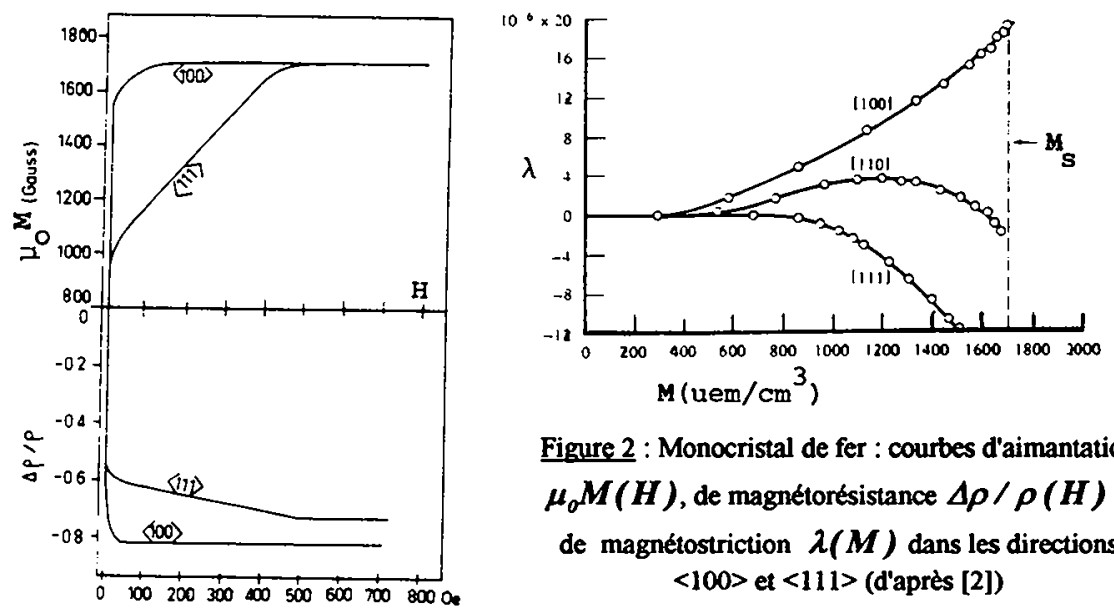

Figure 2 : Monocristal de fer : courbes d'aimantation $\mu_{0} M(H)$, de magnétorésistance $\Delta \rho / \rho(H)$ et de magnétostriction $\lambda(M)$ dans les directions $<100>$ et $<111>$ (d'après [2])

Dans les matériaux massifs, la magnétorésistance à saturation est voisine de $0,3 \%$ pour le fer et $2 \%$ pour le nickel. Les variations maximales sont observées pour le permalloy ( $80 \mathrm{Fe}-$ $20 \mathrm{Ni}): \Delta \rho / \rho \approx 3 \%$ et dans le cas de l'alliage $70 \mathrm{Ni} 30 \mathrm{Co}$, sous forme de film mince (e = $300 \AA): \Delta \rho / \rho \approx 3,8 \%$. Le permalloy qui présente par ailleurs une insensibilité vis à vis des 
contraintes $\left(\lambda_{s} \approx 0\right)$, s'est avéré intéressant pour la réalisation de têtes de lecture (et plus généralement de capteurs magnétiques) magnétorésitive [3].

Certains super-réseaux magnétiques avec un couplage antiferromagnétique entre les couches magnétiques séparées par des couches non magnétiques, présentent une magnétorésistance géante, liée à l'agencement et au couplage des couches. Les effets les plus intenses sont observés sur les multicouches $\mathrm{Fe} / \mathrm{Cr} / \mathrm{Fe}$ et surtout $\mathrm{Co} / \mathrm{Cu} / \mathrm{Co}$ qui conduisent respectivement à $300 \mathrm{~K}$, à des valeurs $\Delta \rho / \rho$ de $20 \%$ et 50 à $60 \%$ [4], ce qui devrait être mis à profit pour la réalisation de têtes magnétorésistives intégrées.

\section{COUPLAGE DIPOLAIRE. ANISOTROPIE DE FORME}

A courtes distances, les interactions dipolaires entre moments magnétiques sont négligeables devant celles d'échange, mais elles dominent à grandes distances. L'action dipolaire dépend de la position relative des points considérés de l'échantillon et de l'existence ou non d'une aimantation en ces points (dimensions finies du matériau). Elle se manifeste par une influence adverse, fonction de la géométrie, que l'on traduit en termes de champ démagnétisant $\vec{H}_{d}=-N \vec{M}$ qui s'oppose à l'imantation $\vec{M}$ du matériau et vient se soustraire à

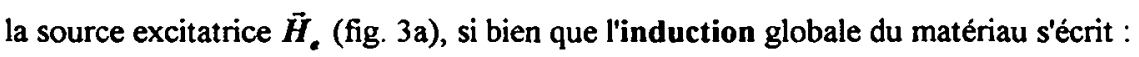

$$
\vec{B}=\mu_{o}(\overrightarrow{\boldsymbol{H}}+\overrightarrow{\boldsymbol{M}}) \text { avec } \overrightarrow{\boldsymbol{H}}=\overrightarrow{\boldsymbol{H}}_{e}+\overrightarrow{\boldsymbol{H}}_{\alpha}
$$

$\mathrm{N}$ est le facteur démagnétisant $(\mathrm{N}=0$ pour le cylindre ou le tore uniformément aimanté, $\mathrm{N}=$ $1 / 3$ pour la sphère, $N=1$ pour une longueur réduite), difficile à déterminer pour les formes quelconques. $\vec{H}_{d}$ a pour effet de "fausser" les valeurs intrinsèques du matériau. Des champs démagnétisants "locaux" peuvent également exister au voisinage d'inhomogénéités et de défauts du réseau, chaque fois que la condition $\vec{\nabla} \cdot \vec{M}=0$ n'est pas satisfaite.

Une conséquence importante du couplage dipolaire est l'anisotropie de forme qui tend à orienter l'aimantation préférentiellement suivant la plus grande dimension de l'échantillon.
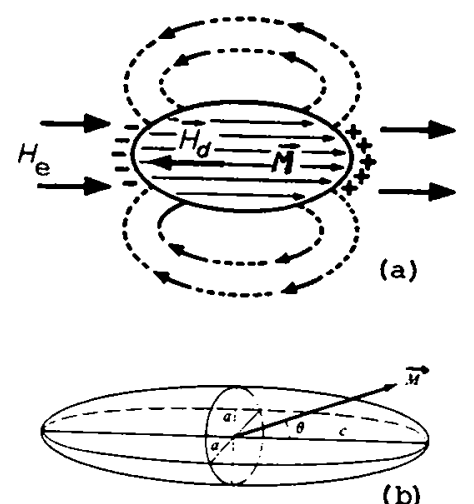

(b)

Figure 3 : (a) Le champ démagnétisant s'oppose au champ applique ; (b) l'anisotropie de forme tend $a$ orienter l'aimantation selon la grande direction $(\theta=0)$

magnétostriction est positive ou négative.
Ainsi dans le cas de l'ellipsoïde allongé de demi axe a (coefficient démagnétisant $\mathrm{N}_{\mathrm{a}}$ ) et $\mathrm{C}\left(\mathrm{N}_{\mathrm{c}}\right.$ ) (fig. $3 \mathrm{~b}$ ), l'axe principal $(\theta=0)$ joue le même rôle qu'une direction de facile aimantation avec la constante d'anisotropie de forme : $K_{s}=0,5 \mu_{o}\left(N_{c}-N_{c}\right) M^{2}$. L'anisotropie de forme est mise à profit dans la fabrication de grains fins destinés à l'enregistrement magnétique, ainsi que d'aimants permanents dont les particules magnétiques sont allongées et alignées dans la direction dans laquelle on souhaite que l'aimantation soit orientée. De même, une couche mince magnétique tend à s'aimanter plus facilement dans son plan que perpendiculairement à son plan. citons :

Parmi les autres formes d'anisotropie possible

L'anisotropie magnétoélastique $K_{\sigma}$ qui tend à privilégier l'aimantation dans la direction parallèle ou perpendiculaire à la tension $\sigma$ selon que la 
L'anisotropie induite $K_{u}$ obtenue en appliquant un champ magnétique au matériau porté à une température inférieure à la température de Curie mais suffisamment élevée pour induire un arrangement atomique ou ordre directionnel.

L'anisotropie d'échange unidirectionnelle, telle celle qui apparaît dans une poudre de cobalt dont les grains sont enrobés par une gaine d'oxyde de cobalt (antiferromagnétique) dont les moments magnétiques interagissent avec ceux de la particule ferromagnétique.

L'anisotropie de surface, décrite par Néel dans le cas de grains très fins [5] et qui est tout à fait actuelle aujourd'hui du fait de son influence prépondérante dans les couches ultraminces (moins d'une dizaine de couches atomiques) et les super-réseaux magnétiques [4]. A la surface des couches magnétiques, l'environnement d'un atome est beaucoup moins symétrique que dans le matériau massif. Cette brisure de symétrie peut privilégier une orientation du moment magnétique perpendiculaire à la surface. Dans de telles couches très minces existe alors une compétition entre l'anisotropie de forme -qui tend à orienter l'aimantation dans le plan du filmet l'anisotropie due aux atomes de surface (ou d'interface avec un film non magnétique), assez nombreux pour orienter l'aimantation dans la direction perpendiculaire aux couches (constante d'anisotropie K). Cette compétition est caractérisée par le facteur de qualité $Q=K / 2 \pi M_{S}^{2}$ qui est supérieure à 1 si l'effet de l'anisotropie de surface domine et inférieur à 1 dans le cas contraire. Des couches minces avec $Q>1$ sont utilisées pour l'enregistrement magnétique haute densité, les mémoires à bulles et pourront certainement être utilisées dans un proche avenir en tant qu'aimants permanents destinés à des dispositifs très miniaturisés.

\section{DOMAINES, PAROIS ET BULLES MAGNETIQUES}

Afin de minimiser son énergie globale, un échantillon ferromagnétique se subdivise spontanément en petits volumes élémentaires, les domaines magnétiques élémentaires ou domaines de Weiss (volume $\approx 10^{-2}$ à $10^{-6} \mathrm{~cm}^{3}$ ). Chaque domaine est aimanté à saturation dans une direction de faible aimantation qui diffère de celle des domaines voisins, si bien que l'aimantation résultante du matériau peut être nulle. Deux domaines adjacents sont séparés par une couche de transition appelée paroi de Bloch, à lintérieur de laquelle l'aimantation tourne progressivement de la direction facile du premier domaine vers celle du deuxième domaine (fig. 4). Dans les structures cubiques avec $K_{1}>0$ (fer), les directions faciles imposent des parois à $180^{\circ}$ et à $90^{\circ}$ alors que pour $\mathrm{K}_{1}<0$ (nickel) elles imposent des parois à $180^{\circ}, 70^{\circ} 5$ et à $109^{\circ} 5$. Dans les substances uniaxes (cobalt), les parois sont à $180^{\circ}$. L'épaisseur d'une paroi résulte de la compétition entre l'énergie d'échange ( $C^{\text {te }}$ d'échange $\mathrm{A}$ ) qui tend à augmenter l'épaisseur et l'énergie magnétocristalline ( $C^{\text {te }}$ d'anisotropie $K_{1}$ ) qui tend à la diminuer.

L'épaisseur $\delta$ et l'énergie $E_{\delta}$ par unité de surface de paroi sont données approximativement par :

$$
\delta \approx \pi \sqrt{A / K_{1}} \text { et } E_{\delta} \approx 2 \pi \sqrt{A K_{1}}
$$

soit pour le fer $\delta \approx 50 \mathrm{~nm}, E_{\delta} \approx 1$ à $2 \times 10^{-3} \mathrm{~J} \cdot \mathrm{m}^{-2}$. Pour des matériaux dotés d'une constante d'anisotropie élevée, tels les aimants permanents $\mathrm{NdFeB}$, l'épaisseur est voisine de $5 \mathrm{~nm}$ et son énergie de $4 \times 10^{-2} \mathrm{~J} \cdot \mathrm{m}^{-2}$. 


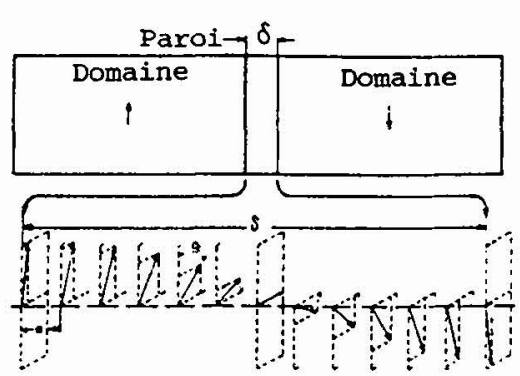

Figure 4: Rotation de l'aimantation à l'intérieur d'une paroi de Bloch à $180^{\circ}$, d'épaisseur $\delta$

Figure 5 : Domaines magnétiques dans un film avec l'aimantation et le champ appliqué perpendiculaires au plan du film : (a) $\mathrm{H}=0$; (b) $\mathrm{H}$ faible; (c) $\mathrm{H}$ suffisant pour créer des bulles magnétiques ; (d) structure d'une bulle.

Figure 6 : Paires de lignes de Bloch (perpendiculaires au film) le long d'une $\Rightarrow$ paroi de Bloch fermé.
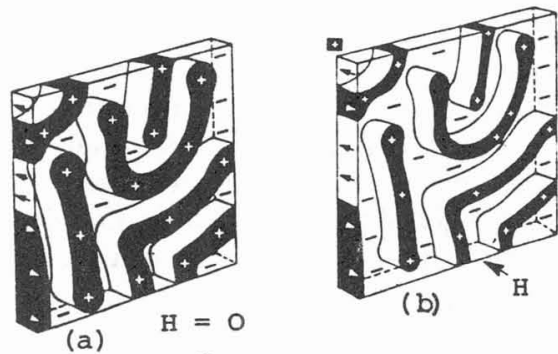

(b)

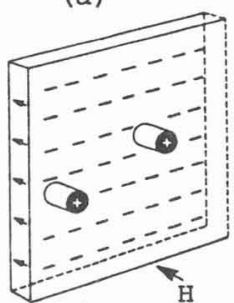

(c)

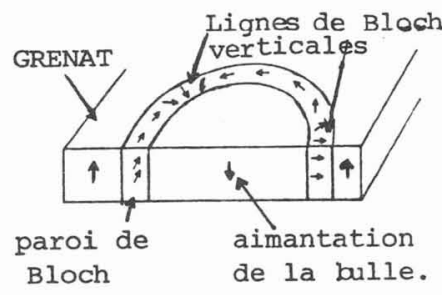

(d)

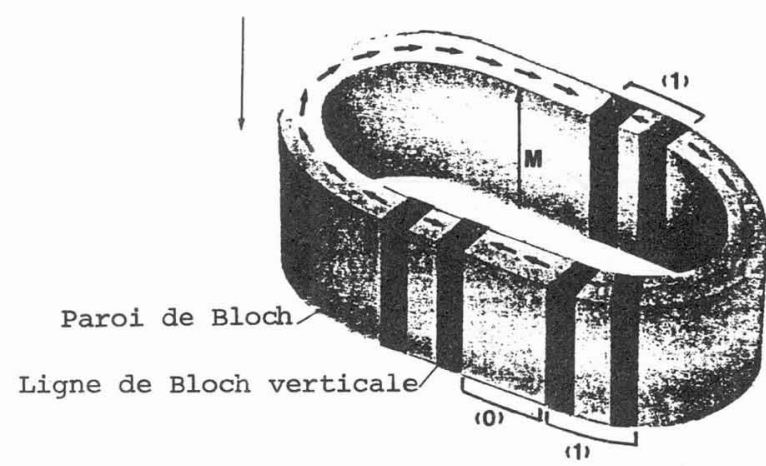

Dans le cas de couches magnétiques suffisamment minces, caractérisées par un coefficient $Q<1$, la rotation de l'aimantation à l'intérieur de la paroi s'effectue parallèlement au plan de la couche : la paroi est dite paroi de Néel. Par contre lorsque $Q>1$, les parois de Bloch à $180^{\circ}$ séparent des domaines en forme de rubans aimantés antiparallèlement. L'application d'un champ magnétique $H$ perpendiculaire aux faces réduit le volume des domaines d'aimantation opposée ; pour une valeur suffisante de $\mathrm{H}$, ces domaines se contractent sous forme de domaines cylindriques, les "bulles" magnétiques (fig. 5a-c). A l'intérieur de la paroi de Bloch délimitant la bulle, l'aimantation est parallèle au plan de la paroi mais peut changer de sens le long de singularités appelées lignes de Bloch verticales (fig. 5d) le long desquelles l'aimantation tourne hélicoïdalement en étant perpendiculaire au plan de la paroi. La dimension des bulles (diamètre $<1 \mu \mathrm{m}$ ) permet de réaliser des mémoires avec une densité de $1 \mathrm{M}$ à $10 \mathrm{Mbit} / \mathrm{cm}^{2}$. Une densité d'information de $250 \mathrm{Mbit} / \mathrm{cm}^{2}$ (théoriquement $1 \mathrm{Gbit} / \mathrm{cm}^{2}$ !) devrait être atteinte en définissant un bit comme la zone comprise entre deux lignes de Bloch verticales (dimension du bit $\approx 0,3 \mu \mathrm{m}$ ). La création d'une paire de lignes de Bloch ainsi que son déplacement le long de la paroi -qui joue le rôle de boucle de stockage (fig. 6)- pouvant être obtenus par l'application de champs magnétiques, il est ainsi possible de réaliser des mémoires à lignes de Bloch verticales, qui devraient passer au stade industriel dans les années à venir $[6,7]$. 


\section{ACTION D'UN CHAMP MAGNETIQUE}

L'application d'un champ magnétique d'intensité croissante sur un matériau ferromagnétique met en jeu successivement des déplacements réversibles puis irréversibles et discontinus de parois : les sauts de Barkhausen (avec croissance des domaines favorablement orientés jusqu'à l'obtention d'une structure monodomaine pour des valeurs de $\mathbf{H}$ suffisantes pour fournir l'énergie nécessaire à la formation de pôles libres sur les faces du matériau) puis des rotations des moments magnétiques dans la direction du champ et atteinte de l'aimantation à saturation $M_{S}$ (fig. 7a). La réduction du champ induit des rotations vers les directions faciles

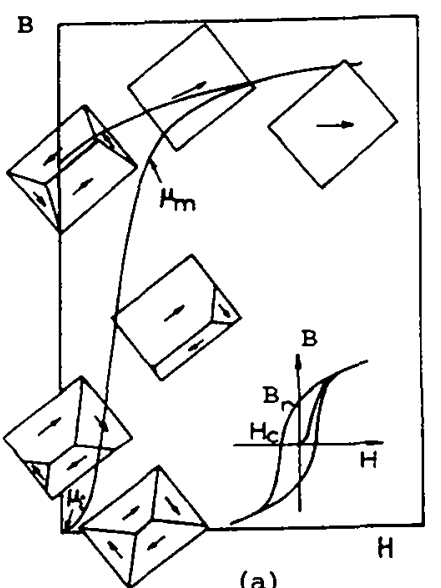

(a)

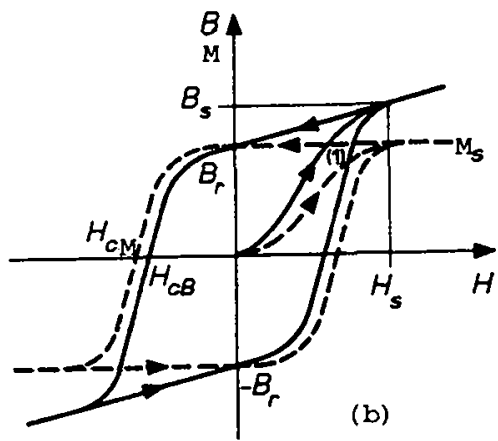

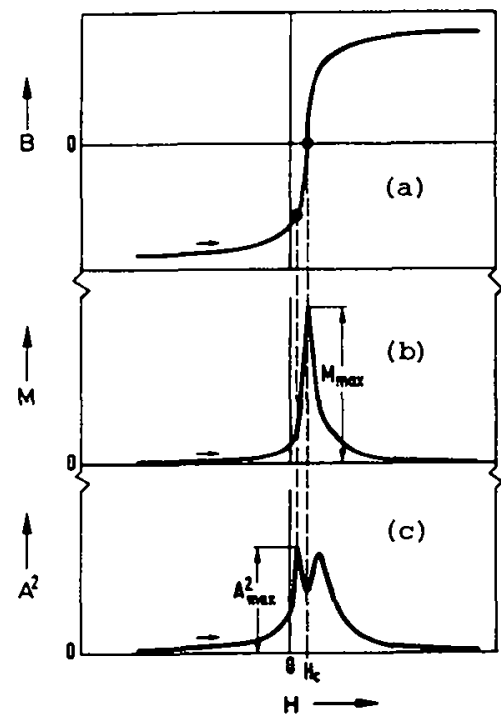

Figure 8 : (a) Branche ascendante du cycle d'induction; Emissions (b) du Bruit de Barkhausen, (c) magnétoacoustique (courbes schématiques).

Figure 7: (a) Evolution de la structure en domaines magnétiques le long de la courbe de première aimantation; (b) courbes de première aimantation $\left(C_{-}\right)$et d'induction $\complement_{\ldots}$ et cycles correspondants

les plus proches de celle de $\mathrm{H}$ puis des germinations de domaines magnétiques. Au point de rémanence $M_{r}=B_{r}$, les courbes d'aimantation et d'induction sont confondues (fig. $7 b$ ). L'application d'un champ opposé diminue l'aimantation et annule successivement :

- l'induction B, pour le champ $\boldsymbol{H}_{C_{B}}=H_{C}$ dit champ coercitif d'induction

- l'aimantation $M$ pour la valeur $H_{C_{H}}$ appelée champ coercitif d'aimantation ou coercitivité intrinsèque. 
On a toujours $\boldsymbol{H}_{C_{M}}>\boldsymbol{H}_{C}$, ces deux valeurs pouvant être généralement confondues sauf dans le cas particulier de certains aimants permanents, tels ceux du type $\mathrm{SmCO}_{5}[\mathrm{~B}=0$ pour $H_{C}=6,4 \times 10^{5}{\mathrm{~A} \cdot \mathrm{m}^{-1}}^{-1}(8 \mathrm{kOe}) ; \mathrm{M}$ vaut encore $0,8 \mathrm{~T}$ et s'annule pour $\boldsymbol{H}_{C_{M}} \approx 12.10^{5} \mathrm{~A} \cdot \mathrm{m}^{-1}$ $(15 \mathrm{kOe})]$. Les grandeurs caractéristiques des cycles d'hystérésis $\mathrm{B}_{\mathrm{r}}, \mathrm{H}_{\mathrm{C}}, \boldsymbol{H}_{C_{M}}$ n'ont de signification intrinsèque pour la définition d'un matériau donné que si l'on effectue le passage par la saturation de l'aimantation. Ces grandeurs dépendent de la température et sont d'autant plus faibles que l'on s'approche du point de Curie.

La mesure de la force électromotrice induite aux bornes d'une bobine entourant un échantillon ferromagnétique soumis à un champ magnétique qui croît de $-\mathrm{H}$ à $+\mathrm{H}$ permet de détecter un bruit, le bruit de Barkhausen. Ce bruit qui est pour une grande part dû aux mouvements discontinus des parois de domaines, passe par un maximum au voisinage du champ coercitif (fig. 8b). Un capteur piézoélectrique mis à la place de la bobine et collé à la surface de l'échantillon permet de capter l'émission d'ondes élastiques, d'orgine magnétostrictive, appelée émission magnétoacoustique. Résultant de la dissipation d'énergie magnétoélastique associée aux réarrangements de la structure en domaines, elle présente deux maximum pour les valeurs de $\mathrm{H}$ qui correspondent aux zones de germination et d'annihilation des domaines (fig. 8c) [8].

\section{LES GRAINS FINS ET LA COERCITIVITE}

Si on diminue la taille d'une particule ferromagnétique, sphérique et bi-domaine, on passe obligatoirement par un diamètre critique $d_{c}$ au-dessous duquel l'énergie de la paroi de Bloch (proportionnelle à $\mathrm{d}^{2}$ ) devient supérieure à l'énergie dipolaire $\left(\sim \mathrm{d}^{3}\right)$. La paroi ne peut plus se former : la particule est monodomaine. Le diamètre critique $d_{c} \approx 18 E_{\delta} / \mu_{0} M_{s}^{2}$, vaut environ 15,40 et $280 \mathrm{~nm}$ respectivement pour une particule isolée de fer, nickel et ferrite de baryum. Dans ces conditions, l'inversion de l'aimantation ne peut se faire que par rotation des moments magnétiques, ce qui peut être mis à profit pour obtenir des matériaux présentant des coercitivités élevées (Fig. 9). A des particules polydomaines correspond une coercitivité assez faible -très faible $\left[<80 \mathrm{~A} \cdot \mathrm{m}^{-1}(1 \mathrm{Oe})\right]$ dans le cas des magnétiques doux-, une grande part de la perte de l'aimantation et de son inversion se faisant par déplacements de parois. Dans le cas de grains monodomaines, deux méthodes peuvent conduire à des coercitivités élevées. Elles sont basées sur :

- l'anisotropie de forme $K_{s}$ : par exemple, des particules de fer monodomaines et sphériques $\left(\boldsymbol{K}_{\boldsymbol{S}}=0\right)$ dont les directions faciles sont alignées parallèlement au champ, présentent une coercitivité due à l'anisotropie magnétocristalline et qui correspond à l'inversion cohérente de

l'aimantation: $H_{C_{M}}=2 K_{1} / \mu_{0} M_{S}=43,2 \times 10^{3} A \cdot m^{-1}$ (540 Oe); mais si on donne à ces particules une forme d'ellipsoïde allongé avec un rapport des axes $c / a=10$, l'inversion de l'aimantation est déterminée par l'anisotropie de forme et $H_{c_{M}}=\left(N_{a}-N_{c}\right) M_{S}=$ $813 \times 10^{3} \mathrm{~A} \cdot \mathrm{m}^{-1}(10,1 \mathrm{kOe})$. En fait, l'expérience montre que dans ce cas la coercitivité, bien qu'élevée, n'excède pas $144 \times 10^{3} \mathrm{~A} \cdot \mathrm{m}^{-1}(1,8 \mathrm{kOe})$ par suite de modes non cohérents de rotation (fanning, curling, buckling) de plus faible énergie, qui abaissent $\boldsymbol{H}_{C_{\boldsymbol{M}}}$. 
- l'anisotropie magnétocristalline élevée : c'est la méthode la plus performante. Ainsi dans le cas des alliages intermétalliques, à base de terres rares $\mathrm{SmCO}_{5}$, la rotation cohérente est déterminée par le champ d'anisotropie $H_{A}: H_{C_{M}}=H_{A}=2 K_{1} / \mu_{0} M_{S} \approx 2 \times 10^{7}$ (250 kOe), si on néglige les effets de champ démagnétisant. En réalité la coercitivité mesurée n'excède pas $20 \%$ de cette valeur, par suite de phénomènes de nucléation liés à la microstructure $[9,14]$.

Enfin si on diminue la taille des particules au-dessous de $10 \mathrm{~nm}$ environ, les forces d'échange ne sont plus suffisantes face aux fluctuations thermiques et le retournement de l'aimantation s'effectue spontanément. Les particules qui ne présentent plus de coercitivité sont dites superparamagnétiques.

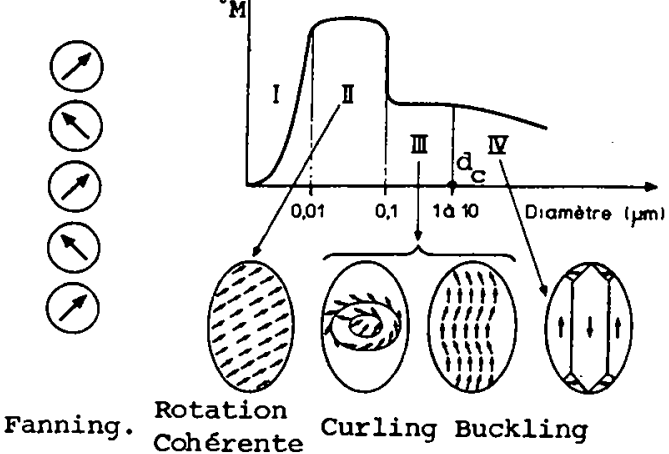

Figure 9: Variation schématique de la coercitivité intrinsèque avec la taille des grains

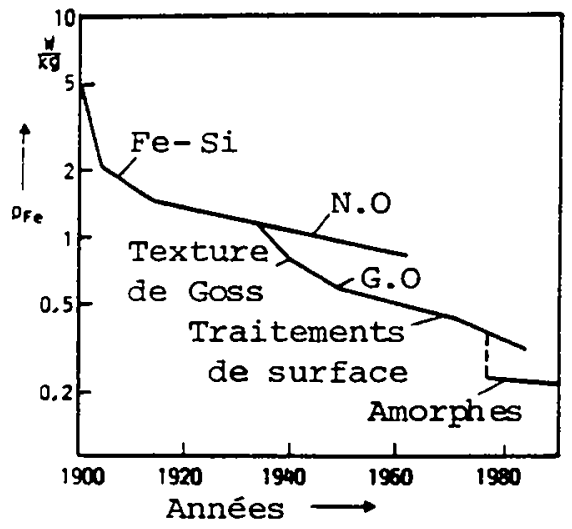

Figure 10: Diminution des pertes magnétiques depuis $1900 ;$ N.O : grains non orientés ; G.O : grains orientés ; d'après [16]

\section{LES MATERIAUX MAGNETIQUES DOUX}

Ils doivent avoir une aptitude à inverser très facilement leur aimantation et à transmettre des variations de flux magnétique (d'où une induction $B_{s}$ et une résistivité électrique $\rho$ élevées). Leur coercitivité est inférieure à $100 \mathrm{Am}^{-1}(1,25 \mathrm{Oe})$ et leur perméabilité relative supérieure à $10^{3}$. Les matériaux les plus doux présentent une bonne pureté et peu de défauts de manière à permettre des déplacements aisés des parois de domaines magnétiques. Les performances optimales sont obtenues lorsque les constantes d'anisotropie magnétocristalline et de magnétostriction sont à la fois proches de zéro, comme c'est le cas pour lalliage $\mathrm{Fe}-5,4 \% \mathrm{Al}-9,6 \% \mathrm{Al}$ (masse) (Sendust : $B_{s} \approx 1 \mathrm{~T}, \rho=85 \mu \Omega . \mathrm{cm}, H_{c}=2 \mathrm{~A}^{-1}$ ), les alliages Fe-(75 à $80 \%) N i\left(78\right.$ permalloy, mumétal, supermalloy ...: $B_{s} \approx 0,8 \mathrm{~T}, \rho \approx$ $60 \mu \Omega$.cm, $\left.\mathrm{H}_{\mathrm{c}}<1 \mathrm{~A} \cdot \mathrm{m}^{-1}, \mu_{\mathrm{r}}(\max ; 50 \mathrm{~Hz})>10^{5}\right)$ et certains alliages amorphes à base de

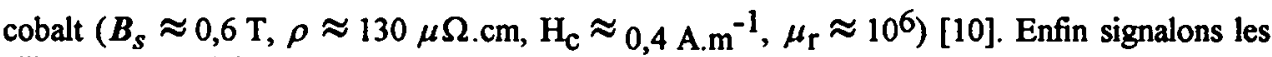
alliages $\mathrm{Fe}_{77,5}(\mathrm{Si}, \mathrm{B})_{22,5}(\mathrm{~atm})$ avec des additions de $\mathrm{Cu}$ et $\mathrm{Nb}$, découverts récemment [11, 12] et qui combinent eux aussi une anisotropie et une magnétostriction faibles. Solidifiés à l'état amorphe, ils présentent après une cristallisation contrôlée (grains c.c de 10 à $12 \mathrm{~nm}$ de diamètre) des propriétés magnétiques comparables à celles des amorphes, mais avec une induction à saturation plus élevée $(1,1$ à $1,3 \mathrm{~T})$. En pratique, tous ces matériaux peuvent avoir des applications variées (blindage, disjoncteurs différentiels, transformateurs d'impulsions et HF, filtres, amplificateurs magnétiques, têtes d'enregistrement et de lecture ...). 
Etant sollicités en régime dynamiques $(50 \mathrm{~Hz}$ à $200 \mathrm{kHz}$ ) dans la plupart de leurs applications, les matériaux doux dissipent de l'énergie sous forme de chaleur. Ces pertes magnétiques qui se décomposent en pertes par hystérésis et pertes par courants de Foucault [13] n'ont fait que s'abaisser au cours des décennies (figure 10) notamment grâce à l'élaboration d'alliages Fe-Si à grains non orientés (avec 1 à $3 \%$ de silicium; utilisés dans les générateurs, moteurs et autres machines tournantes) et à grains orientés ( 3 à $4,5 \% \mathrm{Si}$, avec une texture (110)[001] et des traitements de surface très spécifiques; réservés aux transformateurs) puis à celle des alliages amorphes et nanocristallins. Pour les applications à des fréquences très élevées (10 à $100 \mathrm{MHz}$ ), les ferrites doux $\mathrm{MOFe}_{2} \mathrm{O}_{3}(\mathrm{M}=\mathrm{Mn}-\mathrm{Zn}$ et Ni$\mathrm{Zn})$ sont utilisés du fait de leur grande résistivité $\left(10^{8}\right.$ à $\left.10^{13} \mu \Omega . \mathrm{cm}\right)$ et malgré leur induction à saturation réduite $(0,3$ à $0,45 \mathrm{~T})$.

\section{LES MATERIAUX MAGNETIQUES DURS}

Ils sont utilisés pour la fabrication d'aimants permanents. La fonction première d'un aimant étant de créer une induction magnétique statique, souvent en circuit ouvert, l'aimant est soumis à l'action de son propre champ démagnétisant $\mathrm{H}_{\mathrm{d}}$.Il en résulte que son point de fonctionnement ne se situe pas en $\mathrm{B}_{\mathrm{r}}$ mais en $\mathrm{P}\left(\mathrm{B}_{\mathrm{a}}, \mathrm{H}_{d}\right)$, point d'intersection de la droite de pente $\boldsymbol{p}=\boldsymbol{B}_{a} / \boldsymbol{H}_{d}=\mu_{0}\left(1-N^{-1}\right)$ avec la courbe de désaimantation (2ème quadrant du cycle $\mathrm{B}(\mathrm{H}) ; \mathrm{N}=$ coefficient démagnétisant) (fig. 11). Dans ces conditions, un volume d'aimant $\mathrm{V}_{\mathrm{a}}$ procure dans un entrefer de volume $\mathrm{V}_{\mathrm{e}}$, en l'absence de fuite de flux, une densité d'énergie magnétique : $W_{e}=0,5 B_{a} H_{d} V_{a} / V_{e}$. Un volume d'aimant $\mathrm{V}_{\mathrm{a}}$ donné procure le maximum d'induction dans l'entrefer lorsque l'aimant "travaille" au point optimal $B_{m}, H_{m}$ pour lequel le produit $\mathrm{BH}$ atteint sa valeur maximale $(\mathrm{BH})_{\max }$. Cette valeur, appelée énergie spécifique maximale ou produit d'énergie maximal est une caractéristique première du matériau, au même titre que $\mathrm{B}_{\mathrm{r}}$ et $\boldsymbol{H}_{C_{M}}$, cette dernière caractérisant la résistance à la désaimantation.

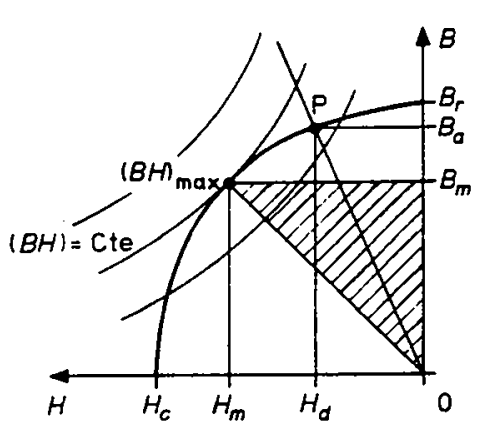

Figure 11 : Courbe de désaimantation et point de fonctionnement optimal $(\mathrm{BH})_{\max }$ d'un aimant

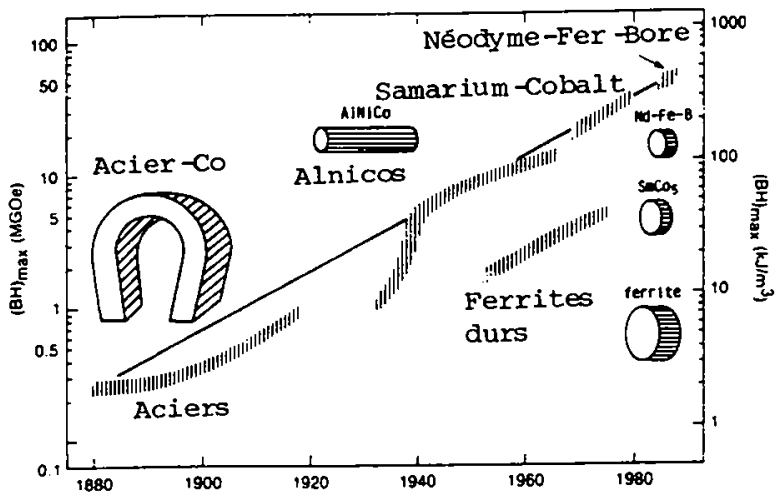

Figure 12 : Evolution de l'énergie spécifique maximale des principaux aimants permanents. Les aimants représentés fournissent la même énergie magnétique ; d'après [17]

L'échelle semi-logarithmique de la figure 12 traduit l'augmentation très rapide subie par $(\mathrm{BH})_{\max }$ depuis la fin du siècle dernier, tandis que les aimants représentés de manière à procurer la même énergie magnétique, schématisent l'évolution de leur puissance. Selon le terme dominant de l'anisotropie on distingue aujourd'hui [14] : 
- les matériaux à anisotropie de forme élevée, tels les alliages $\mathrm{Fe}-\mathrm{Co}, \mathrm{Fe}-\mathrm{Co}-\mathrm{Cr}$ et surtout les AlNiCo qui sont constitués de particules allongées et isolées dans une matrice non magnétique - les matériaux à anisotropie magnétocristalline tels les ferrites de baryum $\mathrm{BaO} \mathrm{Fe}_{2} \mathrm{O}_{3}$ aux caractéristiques relativement modestes mais d'un coût réduit et les alliages à base de terres rares $\mathrm{SmCO}_{5}, \mathrm{Sm}_{2} \mathrm{CO}_{17}$ ainsi que le tout récent $\mathrm{Nd}_{2} \mathrm{Fe}_{14} \mathrm{~B}$ (découvert en 1983) qui est l'aimant le plus puissant actuellement connu $\left((\mathrm{BH})_{\max } \approx 300 \mathrm{kJm}^{-3}\right)$.

\section{LES MATERIAUX POUR LA MEMORISATION DE L'INFORMATION}

Rappelons qu'une mémoire magnétique est essentiellement constituée d'une couche magnétique déposée sur un substrat (bandes, disques, tambours). La création des domaines de mémorisation ( 0 et 1 binaires) dans la couche magnétique s'effectue sans contact, uniquement par le "survol" de la couche par une tête d'écriture (magnétique doux) qui envoie un champ magnétique au rythme rapide des impulsions qu'elle reçoit. La lecture s'effectue de la même manière, sans aucun contact, au moyen d'une tête de lecture (inductive ou magnétorésistive) qui est sensible aux champs magnétiques de fuite qui sortent de la couche magnétique.

Les matériaux pour la mémorisation doivent présenter une aimantation rémanente suffisante (avec $M_{r} / M_{S} \rightarrow 1$ ), une inversion rapide de l'aimantation et une coercitivité suffisamment élevée pour résister aux effaçages dûs aux effets des champs démagnétisants mais cependant limitée pour permettre de futurs enregistrements $\left(\mathrm{H}_{\mathrm{c}} \approx 20\right.$ à $200 \mathrm{kA} \cdot \mathrm{m}^{-1}(250$ à $2500 \mathrm{Oe})$ ). On distingue [15] :

- les couches particulaires constituées de particules monodomaines $(0,3$ à $0,1 \mu \mathrm{m})$ uniformes en taille, morphologie et dopage. Ce sont les particules classiques $\gamma \mathrm{Fe}_{2} \mathrm{O}_{3}, \mathrm{CrO}_{2}$ à anisotropie de forme (acicularité $\mathrm{c} / \mathrm{a}=6$ à $10 ; \mathrm{H}_{\mathrm{c}} \approx 22,5$ à 30,5 kA.m-1). Pour l'enregistrement haute densité ont été mises au point des particules isotropes à anisotropie multiaxiale renforcée dans une direction par dopage avec des ions à anisotropie magnétocristalline élevée, telles Co$\gamma \mathrm{Fe}_{2} \mathrm{O}_{3}\left(\mathrm{H}_{\mathrm{C}}=32\right.$ à $\left.80 \mathrm{kA} \cdot \mathrm{m}^{-1}\right)$ ainsi que des particules avec l'axe facile perpendiculaire au

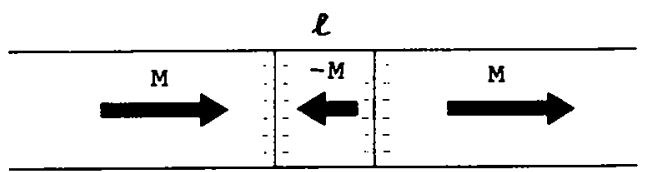

(a)

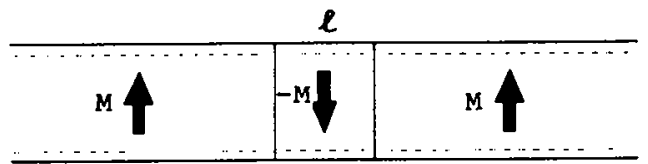

(b)

Figure 13 : Domaine magnétique d'aimantation inverse dans le cas d'un enregistrement (a) longitudinal, (b) perpendiculaire plan du grain (ex: plaquettes hexagonales $\mathrm{BaFe}_{12} \mathrm{O}_{19}(+\mathrm{Co}, \mathrm{Ti}) ; \mathrm{H}_{\mathrm{c}}=25,6$ à $157,6 \mathrm{kA} \cdot \mathrm{m}^{-1}$ ).

- les couches minces uniformes pour enregistrement longitudinal (ex : Co-P; $\mathrm{Q}<$ 1) et perpendiculaire (ex: $\mathrm{Co}-\mathrm{Cr} ; \mathrm{Q}>1$ ). Un bit élémentaire revient à créer un domaine magnétique d'aimantation inverse à celle du reste de la couche avoisinante (fig. 13). Dans le cas de l'enregistrement longitudinal ceci revient à accoler des pôles de même polarité qui donnent lieu à des forces de répulsion: l'énergie magnétique totale augmente avec la densité d'intégration si bien que la taille $\ell$ des domaines (qq $\mu \mathrm{m}$ ) ne peut être trop réduite. Au contraire dans le cas de l'enregistrement perpendiculaire, les domaines adjacents alternent des polarités + et - conduisant à une configuration d'énergie faible : la taille des domaines $(<1 \mu \mathrm{m})$ permet d'obtenir une haute densité d'intégration $\left(10^{7}\right.$ à $10^{8} \mathrm{bits} / \mathrm{cm}^{2}$ ).

- les couches pour mémoires à bulles et à lignes de Bloch verticales : sous forme de films épitaxiés (épaisseur: 2 à $3 \mu \mathrm{m}$ ) de grenat de terres rares ferrimagnétique tel $(\mathrm{YSmLuCa})_{3}(\mathrm{FeGe})_{5} \mathrm{O}_{12}(\mathrm{Q}>1)$ déposés sur un substrat de grenat non magnétique (ex : $\mathrm{Gd}_{3} \mathrm{Ga}_{5} \mathrm{O}_{12}$ ). 
- les couches magnétooptiques dont l'analyse par effet Kerr (réflexion) ou Faraday (transmission) permet de détecter la présence ou l'absence d'un domaine à aimantation inverse. Ces couches doivent présenter une anisotropie uniaxiale perpendiculaire $(Q>1)$, un $\mathrm{H}_{\mathrm{c}} \approx 80$ à $160 \mathrm{kOe}(1$ à $2 \mathrm{kOe})$, une rotation Kerr relativement importante $\left(\theta_{\mathrm{k}}>0,3\right.$ degré) et une température $\mathrm{T}_{\mathrm{c}}\left(\right.$ ferro) ou $\mathrm{T}_{\text {compensation }}\left(\right.$ ferri) proches de l'ambiante $\left(80-200^{\circ} \mathrm{C}\right)$. Elles sont constituées par des films amorphes d'alliages de terres rares et de métaux de transition qui sont soit ferrimagnétiques ( $\mathrm{GdCo}, \mathrm{GdTbCo}$ ) soit ferromagnétiques ( $\mathrm{TbFe}, \mathrm{GdFe}, \mathrm{GdTbFe}$, $\mathrm{GdFeCo}, \quad \mathrm{TbFeCo} .$.$) avec pour \left[\mathrm{TeO}_{1,1}\right]_{90} \mathrm{Ge}_{5} \mathrm{Sn}_{5}$ la possibilité d'enregistrements réinscriptibles. Signalons aussi la découverte récente de multicouches magnétiques $\mathrm{Au} / \mathrm{Co} / \mathrm{Au}$, $\mathrm{Pt} / \mathrm{Co} / \mathrm{Pt}$ présentant une rotation Kerr "géante" $\theta_{\mathrm{k}}>10^{6}$ degrés $/ \mathrm{cm}$ (Co massif : $\theta_{\mathrm{k}}=2 \times 10^{5}$ degrés/cm).

\section{CONCLUSION}

Les améliorations et les innovations apportées ces dernières années aux matériaux magnétiques sont essentiellement basées sur une meilleure connaissance des mécanismes physiques du magnétisme -aux échelles atomique et intermédiaire- ainsi que sur des procédés d'élaboration améliorés ou nouveaux. Les progrès obtenus ont également été stimulés par une demande accrue en vue d'applications toujours plus performantes. Cette rapide revue de quelques propriétés de base et des matériaux magnétiques ne peut donner qu'une image très partielle du rôle de ces matériaux, de l'étendue et de la variété de leurs usages ainsi que de leur impact économique.

\section{Références :}

[1] P. Morin, J. Rouchy, E. de Lacheisserie - Phys. Rev. B 16, 3182, (1977)

[2] I.A. Campbell, A. Fert - Ferromagnetic Materials, North-Holland, Amsterdam, vol.3, (1982)

[3] P. Mas - Thèse Doc. Ing. Univ. Sci. et Médic., Grenoble, (1987)

[4] P. Beauvillain et al. - Images de la Physique, Le courrier du CNRS, 77, 72, (1991)

[5] L. Néel - J. Phys. Rad., 15, 225, (1954)

[6] S. Konishi - IEEE Trans. Magn. MAG-19, 1838, (1983)

[7] J. Miltat, A. Thiaville - Images de la Physique, Le courrier du CNRS, 77, 54, (1991)

[8] M. Guyot, V. Cagan, - JMMM, 101, 256, (1991)

[9] K.D. Durst, H. Kronmüller - JMMM, 68, 63, (1987)

[10] G.Y. Chin, J.H. Wernick - Ferromagnetic Materials, North-Holland, Amsterdam, vol. 2, (1980)

[11] Y. Yoshizawa, S. Oguna, K. Yamauchi - J. Appl. Phys., 64, 6044, (1988)

[12] G. Herzer - IEEE, Trans. Magn., 26, 1397, (1990)

[13] J. Degauque - Mém. Etudes Scientifiques Rev. Métall., 68, 5, (1985)

[14] J. Degauque - Matériaux à propriétés magnétiques dures, Techniques de l'Ingénieur, M340 et M341, 1-21, (1988)

[15] G. Bate - JMMM, 100, 413, (1991)

[16] H. Warlimont - IEEE Trans. Mag., 26, 1313, (1990)

[17] J.F. Herbst, J.J. Croat - JMMM, 100, 57, (1991) 